\section{Ambivalente Haltung der Krankenkassen zur HPV-Impfung}

\author{
Seit März 2007 empfiehlt die Ständige Impfkommission (STIKO) die \\ HPV-Impfung aller Mädchen im Alter von 12-17 Jahren. Der Gemeinsa- \\ me Bundesausschuss (G-BA) bestätigte die STIKO-Empfehlung, sodass \\ einer Aufnahme der HPV-Impfung in die Impfvereinbarung mit den \\ Krankenkassen nichts mehr im Wege stand. In der Praxis stößt man \\ jedoch auf Ungereimheiten.
}

A lle gesetzlichen Krankenkassen und Ersatzkassen müssen heute die HPV-Impfung erstatten. Jedoch beträgt die Durchimpfungsrate der Zielpopulation in Deutschland nur ca. $30 \%$. Wenngleich die Gründe für die niedrige Akzeptanz unklar sind, so fällt doch die ambivalente Informationspolitik mancher großer Krankenkassen zur HPV-Impfung auf und lässt am Umsetzungswillen des Impfprogramms zweifeln. Drei Beispiele:

AOK: Bei der Google-Suche „AOK HPVImpfung" erscheint als erster Treffer „HPV-Impfung: sinnvoll oder nicht? (www.aok.de/portale/bundesweit/hpv/). Auf der Homepage der AOK wird den Mädchen bzw. Müttern eine Bewertung und Entscheidungsfindung zur HPVImpfung angeboten. Unter „Kann die Impfung schaden?" wird ausgeführt, dass „8 von 100 gemeldeten Verdachtsfällen von Impfnebenwirkungen schwer sind. Diese Verdachtsfälle können tat-

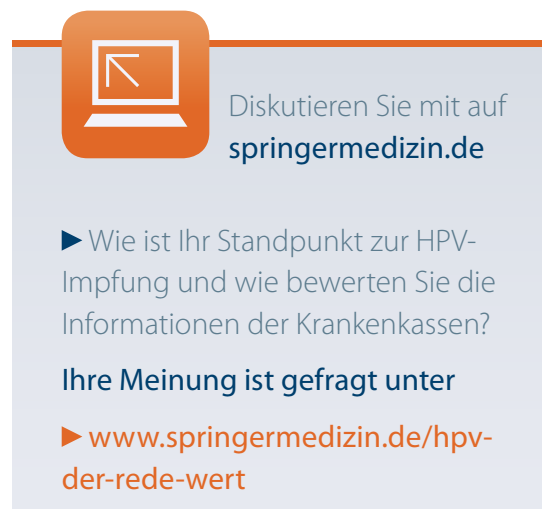

sächlich durch die Impfung verursacht sein oder nur zufällig zur gleichen Zeit auftreten. $\mathrm{Zu}$ ihnen zählen beispielsweise eine Art von Nervenlähmung, Blutgerinnsel und auch Todesfälle. Ein Zusammenhang mit der Impfung konnte nicht in allen Fällen ausgeschlossen werden, er konnte aber auch nicht bewiesen werden. Um seltene Nebenwirkungen und mögliche Langzeitschäden $\mathrm{zu}$ entdecken, muss die Sicherheit über viele Jahre weiter beobachtet werden." Diese Aussagen zur Impfsicherheit dürften Mädchen und deren Mütter erheblich verunsichern. Im Impressum findet sich als Herausgeber der AOK-Bundesverband GbR. Inzwischen sind weltweit mehr als 70 Millionen Dosen der HPV-Impfstoffe verabreicht worden. Die Nutzen/Risiko-Einschätzung verschiedenster Behörden (z. B. Health Protection Agency, Vereinigtes Königreich) ist weiterhin sehr positiv.

Barmer und Techniker Krankenkasse (TK): Bei der Google-Suche: „Barmer HPV-Impfung “ (www.nationales-netzwerk-frauengesundheit.de) und „Techniker Krankenkasse HPV-Impfung“" (www. tk.de/tk/broschueren-und-mehr/krebsfrueherkennung/hpv-impfung/141712) erscheint jeweils eine Veröffentlichung „Früherkennung von Gebärmutterhalskrebs - HPV-Impfung - Information und Erfahrungen - Eine Entscheidungshilfe“, die das Nationale Netzwerk Frauen und Gesundheit in Kooperation mit Barmer bzw. TK herausgegeben hat. In dieser Informationsbroschüre steht zur HPV-Impfung u.a. „In den Zulassungsstudien tra-

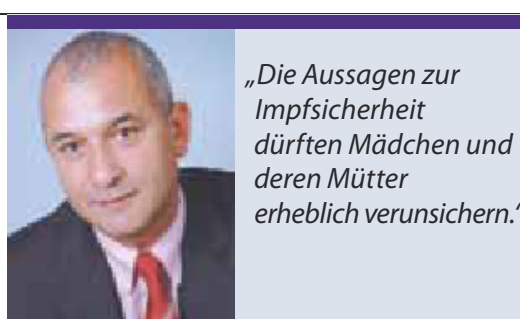

Prof. Dr. Tino F. Schwarz

Facharzt für Laboratoriumsmedizin, Medizinische Mikrobiologie und Infektionsepidemiologie Stiftung Juliusspital Würzburg

ten mittelschwere und schwere Zellveränderungen am Gebärmutterhals bei geimpften Frauen um etwa 20\% seltener auf als bei nicht geimpften (FUTURE II, 2007)."Nicht erwähnt wurde jedoch, dass es sich bei diesen $20 \%$ um die Intentionto-treat-Kohorte handelte und nicht um HPV-naive Frauen. Bei HPV-naiven Frauen, die der primären Zielgruppe der STI$\mathrm{KO}$-Empfehlung entsprechen, war die Wirksamkeit der HPV-Impfstoffe in den klinischen Studien nahezu 100\%. Auf diese hohe Wirksamkeit der Impfstoffe wird jedoch nicht eingegangen. Zur Sicherheit des Impfstoffs wird ausgeführt, dass „auch wenn der Impfstoff vor der Zulassung an 20.000 Frauen getestet wurde, kann die langfristige Verträglichkeit noch nicht abschließend beurteilt werden“. In der Broschüre überwiegen Negativismen wie „Wo kann ich Impfprobleme melden?", „EU fordert Dokumentation der Wirksamkeit [...] nur so könne man langfristig den Nutzen und Schaden der HPV-Impfung überhaupt beurteilen“. Wer aber ist das Nationale Netzwerk Frauen und Gesundheit? Der Blick auf die Homepage dieses Netzwerks dokumentiert eine deutliche negative Einstellung zur HPV-Impfung. Es ist unverständlich, warum Barmer und TK eine Informationsbroschüre zur HPVImpfung bei ausgewiesenen Gegnern der HPV-Impfung in Auftrag gegeben haben und damit die Impfempfehlung der STIKO und die Schutzimpfungsrichtline des G-BA unterlaufen. Über die Beweggründe kann nur spekuliert werden. Dieses Vorgehen widerspricht jedoch dem Auftrag der Krankenkassen und torpediert das Präventionsprogramm. Am schlimmsten ist aber, dass Mädchen und Mütter verunsichert werden, was mit zur Ablehnung der HPV-Impfung beiträgt. Dies scheint offensichtlich von den großen Krankenkassen gewollt zu sein.

Prof. Dr. Tino F. Schwarz 